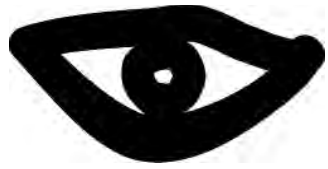

\title{
Praxe no Ensino Superior - Um estudo comparativo entre instituições
}

\author{
Susana Pinho Botelho*, Maria Mendes*, Suzana Nunes Caldeira **, Osvaldo Silva **, Maria José Martins*** \& Célia \\ Barreto Carvalho **** \\ *Universidade dos Açores, Portugal, ** Universidade dos Açores - CICS.NOVA.UAçores, Portugal, *** Instituto Politécnico de \\ Portalegre, Portugal, **** Universidade dos Açores e Centro de Investigação do Núcleo de Estudos e Intervenção Cognitivo- \\ Comportamental, Portugal
}

\begin{abstract}
Resumo
O atual estudo visa compreender o modo como estudantes de duas instituições de ensino superior português percecionam a sua relação com a praxe académica. Participaram no estudo 456 estudantes do $1^{\circ}$ Ciclo de Estudos, da Universidade dos Açores e do Instituto Politécnico de Portalegre. Os dados foram recolhidos através da escala de "Avaliação das Situações de Bullying nas Praxes do Ensino Superior”. No seu conjunto, os resultados permitem presumir que os estudantes do Instituto Politécnico relatam ter melhor relação com praxe académica do que os da Universidade dos Açores.
\end{abstract}

Palavras-chave: Grupo e Processos Interpessoais; Mediação e Resolução de Conflitos; Motivação e Emoção

\section{Introdução}

Nos dias que correm o ingresso no ensino superior começa a ser um passo comum na vida dos jovens portugueses e das respetivas famílias. $\mathrm{O}$ prolongamento da escolaridade obrigatória, primeiro para nove (Lei n. ${ }^{\circ} 46 / 86$ de 14 de outubro) e depois para doze anos (Dec. Lei n. ${ }^{\circ}$ 85/2009) e a estratégia difundida pela Comissão Europeia de incentivo ao conhecimento (Comissão Europeia 5.3.COM 130 final, 2014), muito provavelmente, são fatores que têm concorrido para este acontecimento.

Apesar desta extensão vertical dos estudos se afigurar um percurso natural para muitos jovens, em termos pessoais, a entrada no ensino superior ainda constitui uma etapa muito significativa nas suas vidas (Agante, 2009). Existe a necessidade de adaptação a um novo ambiente de ensino-aprendizagem, em termos do confronto com métodos e materiais de trabalho menos familiares, e também a necessidade de adaptação a um novo ambiente social que, em alguns casos, apresenta exigências no domínio da gestão de vida e, regra geral, o estabelecimento de relações com novos pares.

Assim, embora a decisão de ingressar no ensino superior seja uma resolução do próprio, não deixa de constituir um elevado desafio (Diniz \& Almeida, 2006; Soares, Francischetto, Dutra, Miranda, Nogueira, Leme, Araújo \& Almeida, 2014) no respeitante a mudanças ao nível das aprendizagens académicas, pessoais (Costa, 2008) e de vida, com implicações para a assunção de níveis de autonomia e de iniciativa diferentes daqueles a que o jovem estava habituado (Costa, 2008; Figueira, 2013). Assim, esse ingresso envolve um compromisso e uma responsabilização a nível académico, pessoal e social relativamente às novas experiências e oportunidades oferecidas pela instituição (Seco, Pereira, Dias, Casimiro \& Custódio, 2007) e pelas recentes circunstâncias de vida.

Neste novo cenário de vida, o grupo de pares desempenha um importante papel ao proporcionar experiências emocionais positivas através da aceitação e do reconhecimento dos recém-chegados. Eventualmente por intuírem a importância do grupo de iguais, desejarem a afirmação do seu novo estatuto - o de estudante de ensino superior - e conquistarem o sentimento de identidade grupal, os recém-chegados ao ensino superior encontram nos colegas de anos mais avançados uma referência pertinente. Por sua vez, os estudantes experientes, desenvolvem anualmente momentos que marcam o processo de entrada dos mais novos na instituição. As praxes académicas podem configurar um desses momentos.

São já alguns os relatos de instituições do ensino superior com práticas de 'praxe solidária', isto é, o desenvolvimento de atividades diversas, de índole humanista e participação cívica, que visam promover a integração dos novos estudantes e favorecer o seu bem-estar. Nesse contexto encontram-se, por exemplo, atividades como doação de sangue, na área da saúde, a organização de jogos e campeonatos, no domínio do lazer e desporto, performances em música e teatro, no domínio da cultura, a doação de livros, ligada à educação, o cultivo de plantas e a promoção de reciclagem, referentes ao ambiente, ou doação de roupa, alimentos e brinquedos, no campo do apoio social (Mascarenhas, et al., n.d.).

No entanto, a maioria da literatura que se encontra sobre a praxe académica no ensino superior (e.g., Knutson, Akers, Ellis \& Bradley, 2011; Mikell, 2014) parece caraterizar negativamente esse acontecimento, em sentido análogo ao trazido por Allan e Madden (2008). Estes autores descrevem a praxe como um conjunto de atividades a que os iniciantes são submetidos, independentemente da sua vontade, e que têm um caráter humilhante, ultrajante e abusivo, podendo mesmo fazer perigar a vida dos que a elas são sujeitos. Neste enquadramento, a praxe tem despertado preocupações nas instituições universitárias decorrentes das consequências negativas e dos efeitos maléficos causados nos estudantes (Groves, Griggs \& Leflay, 2011; Rios, 2014).

Pelo exposto observa-se, então, que a praxe académica aparece relatada na literatura científica com 
uma dualidade de sentidos, por um lado integradora e por outro injuriosa. No entanto, os estudantes parecem valorizar a dimensão da integração e desvalorizar a dimensão da injúria. Retomam-se os trabalhos de Allan e Madden (2008), para exemplificar esta ideia, atendendo à grande dimensão do estudo efetuado por estes autores, nos E.U.A.. Assim, Allan e Madden (2008) trabalhando com 11.482 estudantes e vários estabelecimentos de ensino superior, concluíram que, muitas vezes, os estudantes ignoram os comportamentos abusivos na praxe ou os explicam como sendo brincadeiras inofensivas, que veem a praxe com mais efeitos positivos do que negativos e, ainda, que a praxe é referida como um meio de promoção de um vínculo ao grupo. Os mesmos investigadores também concluíram que a maioria dos estudantes que experienciou comportamentos abusivos na praxe afirmou não se sentir maltratada/humilhada. Sendo genuíno este relato dos estudantes, parece que eles aceitam os comportamentos de praxe, não denunciando as humilhações e as agressões que colocam em perigo a sua saúde e segurança, com a intenção de conseguirem pertencer ao novo grupo (Nirh, 2014; Allan \&Madden,2008; Waldron \& Krane, 2005; Phillips, 2013). A vergonha (Finkel, 2002) e/ou o medo da rejeição (Waldron \& Krane, 2005) parecem facilitar a subordinação a essas práticas abusivas.

Em Portugal, sobretudo nestes últimos anos, têm sido noticiados diversos episódios violentos ocorridos no âmbito das praxes académicas, com consequências muito graves. Um dos mais mediáticos ocorreu em dezembro de 2013 na praia do Meco, onde sete jovens presumivelmente envolvidos em praxes académicas foram arrastados por uma onda, tendo seis dos mesmos sucumbido à força do mar (Diário de Notícias, 15/12/2013). Também em 2013, em setembro, foi reportada a morte de uma estudante que havia sido hospitalizada vários meses antes, devido a uma situação de paragem cardiorespiratória, quando estava envolvida em atividades de praxe numa instituição de ensino superior do sul do país (Correio da Manhã, 04/09/13). Mas já antes, em 2009, uma Universidade do norte de Portugal havia sido condenada em Tribunal, em virtude da prática de praxe violenta sobre um estudante de Arquitetura, ocorrência que conduziu à sua morte (Correio da Manhã, de 26/9/2009). A gravidade dos acontecimentos ocasionaram a emissão de um conjunto de recomendações para as instituições de ensino superior por parte do órgão que as tutela (Secretaria de Estado do Ensino Superior, 2014), bem como bem como a criação de um site e a difusão de flyers informativos dirigidos aos estudantes (Ministério da Educação e Ciências, 2014).

No entanto, investigações recentes sobre este tópico têm informado que os estudantes portugueses, de certo modo à semelhança dos estudantes dos E.U.A., parecem não rejeitar a praxe. Por exemplo, na investigação de Dias e Sá (2013), a entrevista a 30 caloiros de Engenharia Eletrotécnica e de Computadores, de um universo de 43 , deu a conhecer que quase metade (47\%) dos entrevistados considerou que a praxe visa facilitar as relações entre colegas e o estabelecimento de novas amizades. Numa outra investigação realizada por Pimentel, Mata, e Pereira (2012), com 273 estudantes da
Escola Superior de Saúde de Bragança, que frequentavam os $1^{\circ}$ e $2^{\circ}$ anos em diferentes cursos na área da saúde, com idade em torno dos 20 anos, encontrou-se que cerca de dois terços dos respondentes concorda com a ideia de que a praxe ajuda os novos estudantes a conhecerem e relacionarem-se com os colegas e a instituição de ensino através de atividades divertidas e num clima de entusiamo. Num outro estudo realizado por Martins, Caldeira, Silva; Botelho e Mendes (no prelo), com uma amostra correspondente a $62.57 \%$ da população estudantil da Escola Superior de Educação de Portalegre, com média de idades de 22.9 anos, foi pedido aos estudantes para expressarem o tipo de participação que haviam tido nas praxes e que as caraterizassem utilizando dois adjetivos à sua escolha. Apenas 9,57\% dos respondentes afirmaram não participar nas praxes, por se declararem anti praxe, e sensivelmente metade dos respondentes caraterizou estas atividades de forma positiva, divertida e favorecedora da sua integração no ensino superior.

Diante desta aparente discrepância entre a ideia de praxe como ritual abusivo e violento ou enquanto diversão e fomento de camaradagem, conhecer melhor o modo como os estudantes dizem relacionar-se com as praxes. Trabalhou-se com estudantes de duas instituições relativamente distanciadas dos grandes centros urbanos: uma situada na região do Alentejo e outra em território insular.

\section{Método}

Participaram na investigação 456 estudantes do $1^{\circ}$ ciclo de estudos, da Universidade dos Açores (UAc, $\mathrm{n}=247$ ) e do Instituto Politécnico de Portalegre (IPP, $\mathrm{n}=209$ ). Os dados foram recolhidos através da "Escala de Avaliação das Situações de Bullying nas Praxes do Ensino Superior" (EASBES) de Matos, Jesus, Simões e Nave (2010), um instrumento com 15 itens de autoresposta Likert (1. total desacordo a 5. total acordo), organizados em três fatores: Relação Positiva com a Praxe (RPP); Relação Negativa com a Praxe (RNP); Dimensão Social (DS). Pontuações mais elevadas significam melhor relação com a praxe. No tratamento dos dados foram utilizados testes não paramétricos ao nível da inferência estatística, uma vez que a aplicação do teste de aderência de Kolmogorov-Smirnov permitiu concluir pela rejeição da hipótese de os dados provirem de uma população com distribuição normal. A produção de resultados assentou no uso do teste $U$ de Mann-Whitney (teste não paramétrico para o caso de duas amostras independentes). Foi ainda utilizada a Análise em Componentes Principais Categórica (CatPCA) para melhor ilustrar a associação entre os fatores da ESABES e o acordo/desacordo expresso pelos estudantes. A opção por esta técnica teve o intuito de reduzir a dimensionalidade das variáveis medidas em escalas diferentes sem perda substancial de informação.

\section{Resultados}

Para perceber a orientação adotada pelo grupo de inquiridos em relação à praxe, foi calculada a 
percentagem de acordo (posições 4 e 5 da escala de resposta) e de desacordo (posições 1 e 2 da escala de resposta). Tendo em conta o grupo total observou-se que $28.2 \%$ dos respondentes discordam das praxes e $71.8 \%$ manifestam-se a favor das mesmas. Esta repartição é sensivelmente replicada em cada uma das instituições de ensino. No IPP $27.3 \%$ dos estudantes discordam das praxes, enquanto $72.7 \%$ se manifesta a favor. Situação idêntica acontece na UAc, onde $29.1 \%$ dos estudantes afirmam discordar das praxes ao passo que $70.8 \%$ declaram estar de acordo.

Apesar de haver similitude entre as duas instituições, traduzida na atitude preponderante de concordância, procurou-se descortinar se existem diferenças no modo como os estudantes de cada uma delas se relaciona com a praxe. Registaram-se diferenças significativas a nível dos resultados globais da escala entre o IPP e a UAc $(p=0.000<\alpha=0.05, U=14992.00$; $\mathrm{p}=0.00$ ), sendo os valores das médias das ordens correspondentes aos alunos do IPP mais elevados (216.22) comparativamente aos dos alunos da UAc (174.99). Deste modo, os estudantes de Portalegre evidenciam melhor relação com a praxe, comparativamente aos dos Açores.

Tendo em atenção a análise de resultados por dimensão, registou-se a existência de diferenças significativas entre os resultados do IPP e da UAc na RPP $\quad(m I P P=243.53 ; \quad m U A=177.98 ; \quad U=14968.5$; $\mathrm{p}=0.000)$ e na DS $(\mathrm{mIPP}=244.73 ; \quad \mathrm{mUA}=197.93$; $\mathrm{U}=18892.5 ; \mathrm{p}=0.000$ ), sempre mais elevados em Portalegre. No que concerne à RNP os resultados não apresentam diferenças significativas entre as duas instituições $\quad(\mathrm{mIPP}=192.0 ; \mathrm{mUA}=209.17 ; \mathrm{U}=18281.0$ $\mathrm{p}=0.113$ ).

No seu conjunto, estes resultados permitem presumir que embora a maioria dos estudantes expresse o seu acordo em relação à praxe académica, os do IPP relatam ter melhor relação com essas práticas do que os da UAc.

Estudaram-se, também, as diferenças entre a instituição do Alentejo e a da região insular, tendo em conta o ano de frequência do curso dos respondentes. Registaram-se diferenças com significado estatístico nos resultados ao nível do $2^{\circ}(\mathrm{mUA}=45.18 ; \mathrm{mIPP}=75.48$; $\mathrm{U}=791.50 ; \mathrm{p}=0.000)$ e do $3^{\circ}$ anos $(\mathrm{mUA}=50.61 ; \mathrm{mIPP}$ =63.88; $\mathrm{U}=1250.5 ; \mathrm{p}=0.037$ ), sendo mais elevados nos estudantes de Portalegre, enquanto no $1^{\circ}$ ano as diferenças registadas não são significativas (mUA=69.38; $\mathrm{mIPP}=68.68 ; \mathrm{U}=2307.0 ; \mathrm{p}=0.917$ ).

Com vista a estudar a associação entre os fatores da ESABES e o acordo/desacordo expresso pelos estudantes das duas instituições de ensino e anos frequentados, e de forma resumir a informação presente nas variáveis originais enunciadas, usou-se uma CatPCA com o método de normalização da variável principal, o qual tem como primordial objetivo perceber a estrutura correlacional das variáveis em estudo. Utilizou-se como critério de retenção das componentes a regra do valor próprio superior a 1 . A informação referente à associação entre as cinco variáveis (fatores da ESABES, instituição de ensino e ano frequentado) foi resumida em duas componentes ortogonais que explicam $63.4 \%$ da variância total das variáveis originais. Na Figura 1 é feita a projeção num gráfico bidimensional das variáveis que contribuem para cada uma das componentes (aquelas cujos pesos são em módulo superiores ou iguais a 0.5 ).

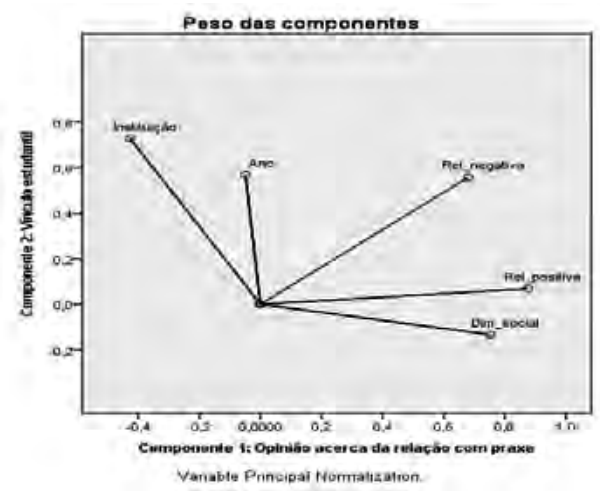

Figura 1 - Pesos das variáveis nas duas componentes

De acordo com os pesos de cada variável em cada componente é possível designar a primeira componente como "Opinião acerca da relação com a praxe” já que os pesos da RPP (0.875), da DS (0.752) e da RNP (0.681), são elevados nesta componente. A segunda componente pode designar-se por "Vínculo Estudantil", dado que os pesos mais elevados obtidos foram nas variáveis Instituição de pertença do aluno (0.730) e Ano frequentado por aquele (0.569).

Com base na Figura 1, verifica-se existir uma forte associação entre os fatores RPP e DS, por um lado, e Instituição e o Ano do curso que os estudantes frequentam, por outro.

O Quadro 3 mostra as quantificações das variáveis utilizadas em cada uma das componentes, apresentando somente as categorias com valores mais elevados e que se encontram em oposição relativamente à componente a que estão associadas.

Quadro 3 - Quantificação das categorias das variáveis em duas componentes.

\begin{tabular}{|c|c|c|c|c|}
\hline & Variáveis & $\begin{array}{r}\text { Quantif } \\
\text { negativas }\end{array}$ & $\begin{array}{c}\text { Quantif } \\
\text { positivas }\end{array}$ & $\begin{array}{l}\text { Variancia } \\
\text { explicada }\end{array}$ \\
\hline \multirow{3}{*}{$\begin{array}{l}-\overrightarrow{0} \\
\stackrel{\Xi}{0} \\
\tilde{0}\end{array}$} & RPP & $\begin{array}{l}\text { Discordo } \\
\text { Totalmente }\end{array}$ & $\begin{array}{l}\text { Concordo } \\
\text { Totalmente }\end{array}$ & \multirow[t]{3}{*}{$39.5 \%$} \\
\hline & RNP & $\begin{array}{c}\text { Concordo } \\
\text { Totalmente }\end{array}$ & $\begin{array}{c}\text { Discordo } \\
\text { Totalmente }\end{array}$ & \\
\hline & DS & $\begin{array}{l}\text { Discordo } \\
\text { Totalmente }\end{array}$ & $\begin{array}{l}\text { Concordo } \\
\text { Totalmente }\end{array}$ & \\
\hline \multirow{2}{*}{ 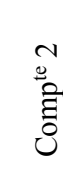 } & Ano & $1 .^{\circ}$ ano & $\begin{array}{l}2 .{ }^{\circ} \text { ano } \\
3 .^{\circ} \text { ano }\end{array}$ & \multirow[t]{2}{*}{$23.9 \%$} \\
\hline & Instituição & $\begin{array}{l}\text { Inst. Pol. } \\
\text { Portalegre }\end{array}$ & Univ. Açores & \\
\hline
\end{tabular}

O mapa percetual, apresentado na Figura 2 dá-nos o posicionamento das categorias das variáveis no espaço definido pelas duas componentes principais retidas, permitindo uma mais fácil visualização das associações entre as categorias dessas variáveis. 


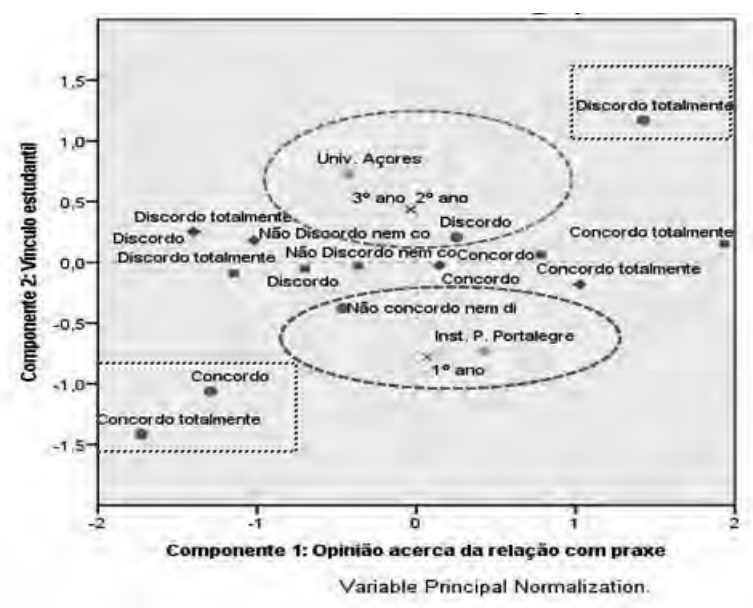

Figura 2 - Mapa percetual definido pelas duas componentes principais retidas.

A componente 1, que explica $39.5 \%$ da variância total, opõe o grupo dos estudantes que expressaram discordância total e discordância com a praxe, aos que declararam concordar e concordar totalmente com essas práticas. A componente 2, que explica $23.9 \%$ da variância total, opõe os estudantes do IPP e que se encontram no $1^{\circ}$ ano, aos que frequentam a Universidade dos Açores e se encontram nos $2^{\circ}$ ano e $3 .^{\circ}$ anos.

A opinião expressa, pelos estudantes, no que respeita à RNP é a que apresenta uma maior dispersão de respostas (ao longo da diagonal secundária), estando estas também relativamente afastadas da Instituição a que aqueles estão vinculados. Realça-se uma elevada proximidade dos alunos do $1^{\circ}$ ano do IPP com a opinião "não concordo nem discordo" no que refere ao fator RNP, evidenciando, neste caso, uma certa ambivalência de opiniões por parte destes alunos. No caso da UAc, relativamente à RNP, são os alunos do $2^{\circ}$ e $3^{\circ}$ ano que expressam uma opinião de menor afeiçoamento à praxe (discorda).

\section{Discussão}

Os resultados encontrados neste trabalho são similares aos obtidos noutros casos (Allan \& Maden, 2008; Pimentel, Mata \& Pereira, 2012). Isto é, os estudantes inquiridos expressaram maioritariamente concordar com os rituais de iniciação na academia (71.8\% do total da amostra manifestou-se a favor). Deste modo, podem tecer-se diferentes conjeturas. Em primeiro lugar pode-se supor que nas instituições em análise não existem práticas abusivas sobre os estudantes do $1 .^{\circ}$ ano. No entanto, também não existem relatos sobre 'praxes solidárias' nestas instituições. Há, ainda, o facto de mais de $25 \%$ dos estudantes afirmar discordar das praxes. Assim, parece ser difícil assumir a ausência de comportamentos coercivos e abusivos. Uma segunda conjetura permite pensar que nas duas instituições os estudantes que responderam em concordância com a praxe podem ignorar ou naturalizar os comportamentos abusivos, explicando-os como brincadeiras esporádicas, com vista a assegurarem a sua integração no grupo. Esta possibilidade, a concretizar-se, levanta algumas preocupações no respeitante ao papel desempenhado pelo grupo de pares, porquanto ele deveria proporcionar experiências emocionais positivas aos recém-chegados e não fomentar a subordinação e sujeição aos veteranos como moeda de troca da aceitação por parte destes. Uma terceira conjetura prende-se com a localização geográfica das instituições, relativamente distantes de grandes centros urbanos. Poder-se-ia presumir que estes estudantes, por se encontrarem em meios mais pequenos, onde os cruzamentos entre as pessoas, na vida quotidiana, têm maior probabilidade de acontecer, as relações de vizinhança tendem a ser mais facilitadas e a situação de anonimato menos plausível, não valorizariam tanto os rituais iniciáticos como um meio para a convivência e integração. Contudo tal não acontece. Esta última conjetura, de apreciação da praxe pelo seu valor em termos de facilitação da integração dos estudantes, ganha maior intensidade no caso de Portalegre. Com efeito, se bem que se tenha encontrado semelhança no respeitante à proporção de acordo/desacordo relativamente às praxes em ambas as instituições, são os estudantes de Portalegre que desenvolvem uma relação ainda mais favorável com a praxe comparativamente aos dos Açores $(\mathrm{U}=14968.5 ; \mathrm{p}=0.000 \quad \mid \mathrm{U}=18892.5$; $\mathrm{p}=0.000$ ), em particular os do $2 .^{\circ}$ e $3 .^{\circ}$ anos. Este facto pode ser indicador de que embora a maioria dos alunos inquiridos estabeleça uma relação de concordância com as atividades da praxe, esta mantém mais atração para os possíveis praxistas em Portalegre do que para o grupo idêntico nos Açores.

Finalmente verifica-se existir uma forte associação entre os fatores RPP e DS e a Instituição e o ano do curso que os estudantes frequentam (Fig. 1). Assim, percebe-se que os estudantes que expressaram uma maior concordância/discordância com a RPP tendem também a expressar uma maior concordância/discordância na DS, isto é darem respostas similares. Percebe-se também que, em geral, são os alunos do $2^{\circ}$ ano e do $3^{\circ}$ ano essencialmente da UAc os que tendem maioritariamente a expressar emoções de desconforto associadas à RNP, enquanto os estudantes do $1^{\circ}$ ano do IPP manifestam uma certa indefinição nas respostas relativamente aos itens deste fator. A partir da redução da informação relacional das 5 variáveis escolhidas (relação positiva, relação negativa, dimensão social, instituição e do ano do curso) em duas componentes principais, que explicam $63.4 \%$ da variância total das variáveis originais, e de acordo com os pesos de cada componente, pode atribuir-se a designação de "Opinião acerca da relação com a praxe" à primeira componente, já que os pesos da relação positiva (0.872) e da dimensão social (0.748) são os mais elevados nesta componente. A segunda pode intitular-se de "Vínculo Estudantil", por resumir a informação do tipo de instituição e do ano do curso, com os pesos da Instituição (0.682) e ano do curso (0.657).

Este é um tópico aparentemente ainda pouco estudado no seio da comunidade académica. Pelo seu impacto na vida de muitos jovens, importa prosseguir esforços de compreensão do papel da praxe, com vista a procurar contribuir para que se fomentem cada vez mais praxes 
'solidárias' favorecedoras da convivialidade e do ajustamento à mudança.

\section{Referências Bibliográficas}

Agante, D. (2009). Comportamentos Relacionados com o consumo de bebidas alcoólicas durante as festas académicas nos estudantes do ensino superior. Dissertação de Mestrado em Saúde Pública. Faculdade de Medicina da Universidade de Coimbra. Coimbra. Disponível

em: https://estudogeral.sib.uc.pt/jspui/bitstream/10316 /13507/1/Tese_mestrado_Diana\%20Agante.pdf

Allan, E., \& Madden, M. (2008). Hazing in view: College students at risck. Initial findings from the national study of student hazing. Acedido em junho de 2014

em http://www.stophazing.org/wp-content/uploads/20 14/06/hazing_in_view_web1.pdf

Chaleta (Ed.) Atās dā III International Conference Learning and Teaching in Higher Education/ I Congresso Ibero-afro-americano de Psicologia.

Comissão Europeia 5.3.COM 130 final (2014). Comunicação da comissão ao parlamento europeu, ao conselho, ao comité económico e social europeu e ao comité das regiões. Acedido em junho de 2014 em http://ec.europa.eu/europe2020/pdf/europe2020sto cktaking_pt.pdf

Costa, S. (2008). Saúde e bem-estar na transição para o ensino superior: Influência dos estilos de vida nos processos de adaptação. Dissertação de mestrado em Psicologia da Saúde. Instituto Politécnico de Beja-Universidade do Algarve. Faro.

Dias, D.,\& Sá, M. (2013). Rituais de transição no ensino superior português: A praxe enquanto processo de reconfiguração identitária. Revista Galego-Portuguesa de Psicoloxía e Educación. Vol. 21, (n. $\left.{ }^{\circ} 1\right)$ Ano $18^{\circ}$, ISSN: 1138-1663. Disponível em http://ruc.udc.es/bitstream/2183/12618/1/RGP 21 2013 art 2.pdf

Diniz, A., \& Almeida, L. (2006). Adaptação à universidade em estudantes do primeiro ano: estudo diacrónico da interação entre o relacionamento com pares, o bem-estar pessoal e o equilíbrio emocional. In Análise Psicológica, 1 (XXIV), pp.29-38. Acedido em junho

de

2015: http://repositorio.ispa.pt/bitstream/10400.12/15 4/1/AP\%2024\%281\%29\%20\%282006\%29\%2029-27. pdf

Figueira, C. (2013). Bem-estar nos estudantes do ensino superior: Papel das exigências e dos recursos percerbidos no contexto académico e das actividades de voluntariado. Tese de doutoramento em Psicologia da Educação. Faculdade de Psicologia-Universidade de Lisboa- Lisboa.

Finkel, M. (2002). Traumatic injuries caused by hazing practices. The American journal of emergency medicine, 20(3), pp. 228-233. Disponível em http://www.njbullying.org/documents/hazing-EDar ticleFinkel2002.pdf

Groves, M., Griggs, G.,\& Leflay, K. (2011). Hazing and initiation ceremonies in university sport: setting the scene for further research in the United Kingdom. In Sport in Society Cultures, Commerce, Media, Politics. Acedido em junho de 2015 em http://dx.doi.org/10.1080/03031853.2011.625287

Knutson, N., Akers, K., Ellis, C.,\& Bradley, K. (2011). Applying the Rasch model to explore new college Sorority and fraternity members - Perceptions of hazing behavior. Disponível em http://www.uky.edu/ kdbrad2/MWERA_Nikki.pd f

Martins, M. J. D., Caldeira, S., Silva, O, Botelho, S. \& Mendes, M. (no prelo). Representações e opiniões dos estudantes de licenciatura sobre as praxes no ensino superior. In III Internacional Conference Learning and Teachinh in Higher Education, I Congresso Internacional Ibero-Afro-Americano de Psicologia. Universidade de Évora.

Mascarenhas, S., Matos, F., Jesus, S., \& Galdino, Z. (n.d.). Diagnóstico e avaliação das praxes ou trotes Um estudo transcultural com universitários de Portugal e do Brasil. Acedido em abril 2014: http://portal.metodista.br/metodista.br/ev/psicologia-d a-saude/anais $1 / 2011 /$ comunicacaooral $/ \operatorname{co} 22 /$ TEXTO $\%$ 20INTEGRAL $\% 20 \% 20$ Escala $\% 20$ Para $\% 20$ Avaliacao \%20Das $\% 20$ Situacoes $\% 20 \mathrm{De} \% 20$ Bullying $\% 20 \mathrm{Nos} \%$ 20Trotes $\% 20$ Do $\% 20$ Ensino $\% 20$ Superior $\% 20 \mathrm{Ap} . \mathrm{pdf} / \mathrm{V}$ iew

Matos, F., Jesus, S., Simões, H., \& Nave, F. (2010). Escala para avaliação das situações de bullying nas praxes do ensino superior. Psyc@w@re, 3 (1). Disponível em: http://www1.ci.uc.pt/ipc/2007-2010/revista/c6944bceb 08cb00930b00b6645171101.pdf

Mikell, T. (2014). Getting away with murder: hazing, hegemonic masculinity, and victimization. Tese de mestrado em Arts in Criminology and Criminal Justice. Universidade da Carolina do Sul, USA. Disponível em: http://scholarcommons.sc.edu/etd/2853

Nirh, J. (2014). Explanations of college students for engaging in hazing. Dissertação para o grau de Doutor em Filosofia com especialização em Ensino Superior, Universidade do Arizona, Arizona, USA. Disponível em https://arizona.openrepository.com/arizona/bitstrea m/10150/321596/1/azu_etd_13324_sip1_m.pdf

Pimentel, M., Mata, M., \& Pereira, F. (2012). Práticas iniciáticas de integração no ensino superior. Um ritual institucionalizado ou um processo de (des) integração? In Atas do V Encontro do CIED-Escola e Comunidade. Lisboa: Escola Superior de Educação de Lisboa, pp. $393-401$.

Phillips, M. (2013). Introduction: Bullying and Hazing as Institutional and Societal Problems. Northeastern University Law Journal,5(1), pp.1-13. Disponível em http://poseidon01.ssrn.com/delivery.php?ID=2200 9012706500009612512208712710606405403808903 7048042010100086081118002071113089093100042 0331220090450470680181270780991180290520780 4303409312511509109100210312400706904412500 1076118094001066019119004070080015117013124 $086001106067029096085104074 \& E X T=$ pdf $\&$ TYPE $=$ 2 
Rios, J. (2014). A case study in promising practices in anti-hazing education training for fraternity advisors. A dissertation in partial fulfillment of the requirements for the degree doctor of education of the USC Rossier School of Education University of Southern California, USA.

Disponível em http://digitallibrary.usc.edu/cdm/ref/collection/p15 799coll3/id/408302

Seco, G., Pereira, I., Dias, I., Casimiro, M., \& Custódio, S. (2007). Construindo pontes para uma adaptação bem-sucedida ao ensino superior: Implicações práticas de um estudo. Comunicação apresentada no $I X$ Congresso da Sociedade Portuguesa de Ciências da Educação. Madeira. Disponível em http://hdl.handle.net/10400.8/18
Soares, A., Francischetto, V., Dutra, B., Miranda, J., Nogueira, C., Leme, V., Araújo, A., \& Almeida, L. (2014). O impacto das expectativas na adaptação acadêmica dos estudantes no ensino superior. Psico-USF, 19, (1), pp. 49-60. Acedido em junho de 2015: http://dx.doi.org/10.1590/S1413-827120140001 00006

Waldron, J., \& Krane, V. (2005). Whatever it takes: Health compromising behaviors in female athletes. QUEST, 57, pp. 315-329. Disponível em http://www.humankinetics.com/acucustom/sitena me/Documents/DocumentItem/4936.pdf 